Results: Patients with a high internal health locus of control (HLCint 13.1 vs. 9.1) had less pain (numeric rating scale $0-10$ ), less flares, a better mental and physical health related quality of life, lower disease activity and less fatigue. Patients with a high external 'doctor'-related health locus of control (HLCdoc 10.7 vs. 7.2) were older, had more co-morbidities, more disease damage and received more frequently an immunosuppressive therapy. No significant differences were found between the patients with a high external 'chance'-related HLC compared to the lower scoring patients (HLCcha 11.1 vs. 6.3). Participants with a high external 'doctor'-related HLC had a more threatening view on their illness and a better adherence to medication (high adherence in $78.6 \%$ vs. $59.4 \%$ ). Participants with a high internal HLC perceived their disease significantly less threatening. Higher education levels (school education, further education) went along with a decrease of external 'doctor'-related HLC (HLCdoc).

Conclusions: Health locus of control has a significant impact in patients with SLE. Depending on the HLC different disease characteristics, treatments, levels of medication adherence and illness perception were noticed. Holistic care needs to consider the impact different HLCs may have. The direction of causality cannot be proved beyond reasonable doubt in this cross-sectional analysis. Hence additional longitudinal studies are necessary.

Acknowledgements: The LuLa-study is supported by unrestricted grants from GlaxoSmithKline and UCB Pharma.

Disclosure of Interest: G. Chehab Grant/research support from: GlaxoSmithKline and UCB Pharma for performing the LuLa-study, J. Richter Grant/research support from: GlaxoSmithKline and UCB Pharma for performing the LuLa-study, R. Brinks: None declared, R. Fischer-Betz Grant/research support from: GlaxoSmithKline and UCB Pharma for performing the LuLa-study, B. Winkler-Rohlfing: None declared, M. Schneider Grant/research support from: GlaxoSmithKline and UCB Pharma for performing the LuLa-study

DOI: 10.1136/annrheumdis-2017-eular.3720

\section{AB1128 HELICOBACTER PYLORI IN SYSTEMIC LUPUS ERYTHEMATOSUS ITS ASSOCIATION WITH ENDOSCOPIC AND HISTOPATHOLOGIC FINDINGS}

1. Arroyo Prado ${ }^{1}$, J.A. Velarde Ruiz Velasco ${ }^{2}$, C.E. Gomez Lopez ${ }^{1}$, S. Gutierrez Ureña $^{1}$, G. Martinez Bonilla ${ }^{1}$, V. Gonzalez Diaz ${ }^{1}$, J.I. Espejo Pascencia ${ }^{3}$, S. Cerpa Cruz ${ }^{1}$. ${ }^{1}$ Rheumatology; ${ }^{2}$ Gastroenterology; ${ }^{3}$ Pathology, Fray Antonio Alcalde Hospital, Guadalajara, jalisco, Mexico

Background: Helicobacter pylori $(\mathrm{Hp})$ is a Gram-negative bacteria and the cause of most of the chronic gastric infections and its prevalence is above $50 \%$ worldwide. This infection is a well-known risk factor to gastric MALT Iymphoma but also could be the trigger of several autoimmune diseases such as immune thrombocytopenic purpura and systemic lupus erythematosus

Objectives: To determine the frequency of $\mathrm{H}$ pylori in systemic lupus erythematosus patients (SLE).

Methods: A cross-sectional study was done in patients who fulfilled the 2012 SLICC criteria for SLE and were willing to sign the informed consent to be subjected to endoscopic procedure. The tissue sample was analyzed by pathologist. We used mean and standard deviation to describe the data, to compare both groups Student $t$ test was done and for continuous variables we used chi-square; the correlation analysis was performed with

Results: Twenty two SLE patients were included and we chose a control group from database of endoscopic clinic with diagnosis of functional dyspepsia. Mean age of study group was 31 vs 48 year-old, $95 \%$ were women, $32 \%$ with immunosuppressant and $95 \%$ were taking antimarials. The frequency of $\mathrm{Hp}$ in SLE patients was $60 \%$, dsDNA and anti-Ro autoantibodies were associated with the presence of $\mathrm{Hp}$; the study group had more frequency of nodular gastritis, metaplasia and dysplasia. Seventy per cent of patients had less than 5 years of diagnosis.

Conclusions: We found a high frequency of $\mathrm{H}$ pylori infection in patients with SLE. Metaplastic and dysplastic changes were also more prevalent in the SLE group. Our data suggest that $\mathrm{Hp}$ infection took place in early stages of disease.

[1] Daniel S Smyk, Andreas L Koutsoumpas, Maria G Mytilinaiou, Eirini I Rigopoulou, Lazaros I Sakkas, Dimitrios P Bogdanos. Helicobacter pylori and autoimmune disease: Cause or bystander. World J Gastroenterol 2014 January.

[2] Sarfaraz Ahmed Hasni Role of helicobacter pylori infection in autoimmune diseases. Curr Opin Rheumatol. 2012 July; 24(4): 429-434.

Acknowledgements: no clinficts of interest.

Disclosure of Interest: None declared

DOI: 10.1136/annrheumdis-2017-eular.4166

\section{AB1129 A SYSTEMATIC REVIEW ON PREVALENCE OF BACK PAIN AND SPONDYLOARTHRITIS BASED ON COPCORD STUDIES}

J. Moreno-Montoya ${ }^{1}$, J. Rodriguez-Amado ${ }^{2}$, R. Burgos-Vargas ${ }^{3}$,

I. Pelaez-Ballestas ${ }^{3} .{ }^{1}$ Universidad del Bosque, Bogota, Colombia; ${ }^{2}$ Research

Associate of South Florida, Miami, United States; ${ }^{3}$ Rheumatology Unit, Hospital General de Mexico, Mexico, Mexico

Objectives: To determine, through a systematic review and meta-analysis, the prevalence of back pain (BP) and spondyloarthritis $(\mathrm{SpA})$ in the adult general population and explore the heterogeneity between studies in and out Latin America (LATAM).

Methods: MEDLINE, Embase, BIREME, LILLACS and Web of Science were searched using a strategy combining key words and related database-specific subject terms to identify relevant cross-sectional studies based on COPCORD methodology published since 2006. Included articles were assessed for risk of bias and quality based on the STROBE statement. Prevalence figures for BP and SpA (European Spondyloarthropathy Study Group criteria) were analyzed according to female percentage of sampled individuals, mean age and sample size. A mixed effect model was used to obtain the combined prevalence and a meta-regression to estimate the effects of these variables. Prevalence stratified values were obtained according to its geographical location.

Results: 44 out of 127 papers in English, Spanish or Portuguese were selected. Of them, 16 contained BP or SpA prevalence data. Estimates for any Spa prevalence ranged from $0,1 \%$ to $2 \%$, with an average of $0,3 \%(95 \% \mathrm{Cl}: 0,01 \%>0,05 \%)$. The random-effects pooled prevalence was $0,18 \%(0,06 \%>0,36 \%)$. The prevalence of BP was $6.54 \%(3.8 \%>9.2 \%)$ with a pooled value of $5.24 \%(2.6 \%>8.7 \%)$. In both cases the heterogeneity was significant $(p<0.01)$. No effect was associated to SpA heterogeneity, but an increase in the prevalence of BP was associated to sample size (random effect coefficient: $0,045, \mathrm{p}=0.04$ ). The stratified analysis did not show differences in terms of heterogeneity or prevalence for BP (Pooled prevalence for BP: $5.4 \% ; 2.9 \%>8.5 \%, p=0.9)$; on the contrary, for Spa, for non-LATAM studies, the pooled proportion was significant bigger (prevalence in LATAM $0.05 \%, 0.01 \%>0.012 \%$; non-LATAM: $0.35 \%, 0.09 \%>0.78 \%, p=0.03$ )

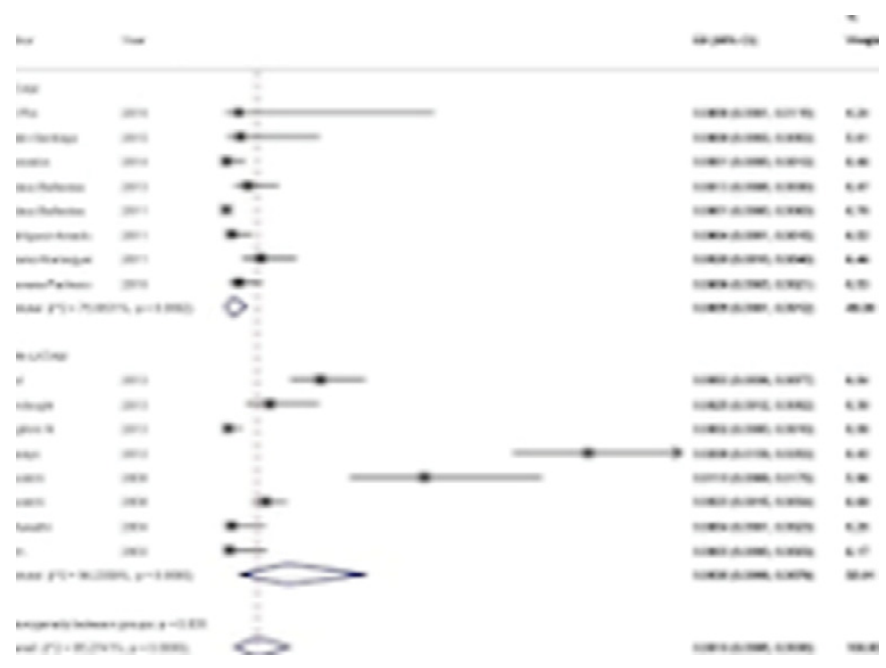

Conclusions: We found significant variations in prevalence across this review. In particular, they related to sample size of BP studies. Similarly, there was a significant variation between LATAM versus other latitudes respect to the prevalence of SpA. The limited number of studies included in this meta-analysis however, prevents clear explanations of the mechanisms underlying these results.

Disclosure of Interest: None declared

DOI: 10.1136/annrheumdis-2017-eular.3916

\section{AB1130 RELAPSE RISK ASSESSMENT IN YOUNG APS PATIENTS WITH PREVIOUS STROKE EVENT USING THE ADJUSTED GLOBAL ANTIPHOSPHOLIPID SYNDROME SCORE (AGAPSS)}

I. Cecchi ${ }^{1}$, M. Radin ${ }^{1}$, K. Schreiber ${ }^{2}$, M.J. Cuadrado ${ }^{3}$, C. Perez-Sanchez ${ }^{4}$, C. Lopez-Pedrera ${ }^{4}$, D. Roccatello ${ }^{5}$, S. Sciascia ${ }^{5} .{ }^{1}$ Department of Clinical and Biological Sciences, Center of Research of Immunopathology and Rare Diseases- Coordinating Center of Piemonte and Valle d'Aosta Network for Rare Diseases, Turin, Italy; ${ }^{2}$ Department of Thrombosis and Haemophilia, Guy's and St Thomas' Hospital; '3 Guy's and St Thomas' NHS Foundation Trust, London, United Kingdom; ${ }^{4}$ GC-5/Rheumatology, IMIBIC/Reina Sofia Hospital/University of Cordoba, Cordoba, Spain; ${ }^{5}$ Department of Clinical and Biological Sciences, Center of Research of Immunopathology and Rare Diseases - Coordinating Center of Piemonte and Valle d'Aosta Network for Rare Diseases, Turin, Italy

Background: The most frequent manifestation of arterial thrombosis in patients affected by Antiphospholipid syndrome (APS) is ischaemic stroke, especially in young adults (less than 50 years old) [1]. Young adults affected by APS are a group of patients at greater risk of developing serious stroke events and recurrences of thrombosis. Therefore, risk stratification in this particular group is crucial, especially in order to prevent a recurrence of ischaemic thrombotic event. Objectives: With the present study we aimed to assess the clinical usefulness of the adjusted Global AntiphosPholipid Syndrome Score (aGAPSS) [3] for risk stratification of thrombosis relapse and/or progression of known ischaemic lesions dectected with Magnetic Resonance Imaging (MRI) in a cohort of young adult APS patients 